\title{
AUTOMAÇÃO DO PROCESSO DE AVALIAÇÃO DE DESEMPENHO DE TRATORES E IMPLEMENTOS EM CAMPO ${ }^{1}$
}

\author{
EVANDRO CHARTUNI MANTOVANI ${ }^{2}$, MICHAEL LEPLATOIS ${ }^{3}$ e RICARDO YASSUSHI INAMASSU ${ }^{4}$
}

RESUMO - Em muitas regiões do Brasil, tratores e implementos agrícolas têm sido usados para dar suporte aos diferentes sistemas de produção, sem uma adequação própria às condições de solo e clima. Para auxiliar os fabricantes e agricultores na solução de problemas em máquinas e implementos agrícolas, há necessidade de verificar o desempenho desses equipamentos e estabelecer condições de testes semelhantes àquelas em que estiveram sendo usados. Este trabalho teve como objetivo avaliar o desempenho de um conjunto trator-implemento agrícola, no campo, utilizando um sistema eletrônico de medições. A potência demandada pelos equipamentos foi calculada indiretamente, com os dados de rotação do motor e consumo de combustível, nas curvas de calibração do trator. O uso de instrumentação eletrônica permitiu que se tivesse uma grande capacidade de trabalho, em razão da facilidade de programação e operação da cadeia de medições. Os resultados de campo obtidos para esforço de tração líquida indicaram tendência de comportamento similar à dos calculados por meio de fórmulas, com uma correlação de 0,996 . A metodologia utilizada nos testes de desempenho operacional do conjunto trator-implemento no campo indicou ser uma ferramenta de bastante utilidade, tanto para os usuários dos equipamentos, quanto para os fabricantes.

Termos para indexação: mecanização agrícola, instrumentação eletrônica, testes de equipamentos.

\section{ELECTRONIC INSTRUMENTATION USE ON FIELD PERFORMANCE TEST OF AGRICULTURAL TRACTORS AND IMPLEMENTS}

\begin{abstract}
Power machinery and implements are being used in many regions of Brazil to support different grain production systems without proper adaptation for working in different soil and weather conditions. To help farmers and manufacters solve problems related to equipments, a performance test needs to be working under a similar field condition. The objective of this research was to evaluate an in-field performance test, using an automatic data acquisition system. The engine power demanded was calculated indirectly, by using the engine rpm and the fuel consumption data on the tractor's calibration curves. The easiness of programming and operating the electronic instrumentation systems allowed to accomplish a great number of performance tests. The results showed very little difference between drawbar force measured and calculated obtaining 0.996 of correlation. The metodology used for in-field performance test showed to be a very powerfull tool to help farmers and the industry better manage their equipments and to improve performance.
\end{abstract}

Index terms: agricultural mechanization, automatic data acquisition, performance tests.

\footnotetext{
${ }^{1}$ Aceito para publicação em 28 de setembro de 1998.

${ }^{2}$ Eng. Agr., Ph.D., Embrapa-Centro Nacional de Pesquisa de Milho e Sorgo (CNPMS), Caixa Postal 151, CEP 35701-970 Sete Lagoas, MG. E-mail: evandro@cnpms.embrapa.br

${ }^{3}$ Eng. Agr., CIRAD/SAR, 73, Rue J.F. Breton - Domaine de Lavalette, 34.000 - Montpellier, França.

${ }^{4}$ Eng. Mecânico, D.Sc., Embrapa-Centro Nacional de Pesquisa e Desenvolvimento de Instrumentação Agropecuária (CNPDIA), Caixa Postal 741, CEP 13560-970 São Carlos, SP.
}

\section{INTRODUÇÃO}

Em muitas regiões do Brasil, equipamentos agrícolas têm a sua utilização comprometida, em razão da sua não-adequação às condições de trabalho. Este fato vem sendo detectado por especialistas da área em contato com agricultores, quando em visita a propriedades agrícolas, dias de campo, seminários e 
outros eventos. Segundo Mantovani (1987), para que um equipamento seja utilizado racionalmente, é necessário conhecer o sistema de manejo de solo que ele vai atender, as características desejáveis que o solo deverá apresentar, a energia consumida e, também, a sua capacidade efetiva de trabalho (ha/h). De maneira geral, os fabricantes têm tentado resolver tais problemas, mas existem poucos dados sistematizados sobre as características do Brasil para utilizá-los como parâmetros de projetos de adaptação e adequação. Os problemas começam a ser estudados por diferentes instituições que trabalham com máquinas agrícolas, tais como universidades, centros de pesquisa e o Serviço de Extensão Rural. Entretanto, pelo fato de o país possuir dimensões continentais, com alta variabilidade regional e com poucos profissionais habilitados para assistência técnica, muitos dos dados podem ser considerados pontuais e isolados do contexto nacional. Mais grave ainda do que essas condições é o fato de que os trabalhos são realizados de forma a resolver os problemas sem que os envolvidos tenham participado da elaboração do projeto da máquina e sem a tão requerida integração de conhecimentos com fabricantes de equipamentos.

Para avaliar o desempenho de equipamentos ou solucionar os problemas causados por eles ao solo ou à planta, é necessário estabelecer condições semelhantes àquelas em que os equipamentos estão trabalhando no campo, e verificar os pontos positivos e negativos durante a operação de campo. Gamero et al. (1986) analisaram o consumo de combustível e a capacidade de campo de diferentes sistemas de preparo periódico do solo, e observaram que a enxada rotativa é o equipamento testado que apresenta maior eficiência de operação e menor consumo de combustível para o preparo do solo por metro cúbico mobilizado. Já o arado é o equipamento que consome a maior quantidade de óleo diesel por metro cúbico do solo mobilizado. Nesta situação, é necessário uma metodologia apropriada e padronizada, para dar suporte aos testes de campo, que não deixem dúvidas da forma como os equipamentos foram testados, e que os dados possam ser utilizados por fabricantes ou instituições em todas as regiões do país. Quanto aos testes de campo sem a utilização de instrumentação eletrônica, a quantidade de área demandada para a sua realização e avaliação de desempenho dos equipamentos é muito grande, o que dificulta a obtenção dos dados e exige a necessidade de realização de muitas repetições. Hoje, com a disponibilidade de instrumentação eletrônica, de custo razoável, montada no trator e implementos, pode-se ter aquisição automática dos dados, propiciando, com isso, maior facilidade na realização dos testes, com melhorias na obtenção de dados mais precisos medidos no campo, e com um conseqüente aumento na quantidade de testes de campo.

O objetivo deste trabalho foi avaliar o desempenho de um conjunto trator-implemento agrícola no campo, por meio de um sistema eletrônico de medições.

\section{MATERIAL E MÉTODOS}

Este trabalho foi desenvolvido na Embrapa-Centro Nacional de Pesquisa de Milho e Sorgo (CNPMS), em Sete Lagoas, MG, em parceria com Centre de Coopération Internationale en Recherche Agronomique pour le Développement (CIRAD), de Montpellier-França, e Embrapa-Centro Nacional de Pesquisa e Desenvolvimento de Instrumentação Agropecuária (CNPDIA), em São Carlos, SP, em 1995.

O método utilizado baseia-se na determinação dos seguintes parâmetros: curva de potência do motor (em laboratório), consumo de combustível, rotação do motor, velocidade de deslocamento, força de tração entre trator-implemento, e profundidade de trabalho, de acordo com Pirot \& Vaitilangom (1987) e Perret et al. (1994).

A cadeia de medições é realizada em três etapas independentes. A primeira é o levantamento indireto da curva de potência do motor. A segunda é a aquisição e o armazenamento de dados no campo, e a terceira é o processamento dos dados obtidos gerando um relatório de desempenho do conjunto trator-implemento-solo.

\section{Levantamento indireto da curva de potência do mo-} tor

O levantamento da curva de potência, aqui denominado calibração do trator, foi feita mediante um banco de ensaio: dinamômetro portátil modelo Nebraska 400, da AW (EUA) com capacidade de até $400 \mathrm{~kW}$, que é ligado à tomada de força. $\mathrm{O}$ princípio de funcionamento é o de um freio mecânico, que testa o trator sob diferentes condições de carga, e que por meio de um torquímetro ao 
mesmo tempo mostra a potência desenvolvida pelo motor. Neste dinamômetro existe um sistema de refrigeração hidráulico para dissipar o calor produzido pelo freio, Liljedahl et al. (1979).

As curvas de calibração indicam a potência indiretamente desenvolvida pelo motor do trator em função do consumo de combustível e da rotação do motor. Nesses testes foram calculados a potência zero, intermediária e máxima em cada intervalo de 100 rotações por minuto. A Fig. 1 mostra as curvas de calibração obtidas com o dinamômetro em um trator Massey Ferguson 292 turbo, com o consumo de combustível em L/hora e a potência na TDP, em kW. O teste foi realizado na Embrapa-CNPMS, em 1995.

\section{Aquisição e armazenamento de dados}

A aquisição de dados é efetuada mediante um conjunto de sensores e um coletor de dados para leitura e armazenamento de dados. A Fig. 2 mostra a posição da caixa de aquisição e de cada um dos sensores usados no conjunto trator-implemento.

Para descrever o processo, serão mostrados, a seguir, os elementos do sistema de medida utilizados no conjunto trator-implemento, assim como o princípio de funcionamento de cada um. Levando-se em consideração os três pontos mais importantes para a programação do data logger ou coletor de dados: memória disponível, característica do coletor ("data-logger") e precisão, o tempo de um segundo foi escolhido para a obtenção de dados, nos testes de campo. Para diminuir o tamanho do arquivo de dados e ao mesmo tempo evitar erros com os dois tipos de sensores (lógicos e analógicos), os dados são acumulados durante intervalos de 5 segundos, e depois, o valor médio é gravado na memória final. Dessa forma, o programa

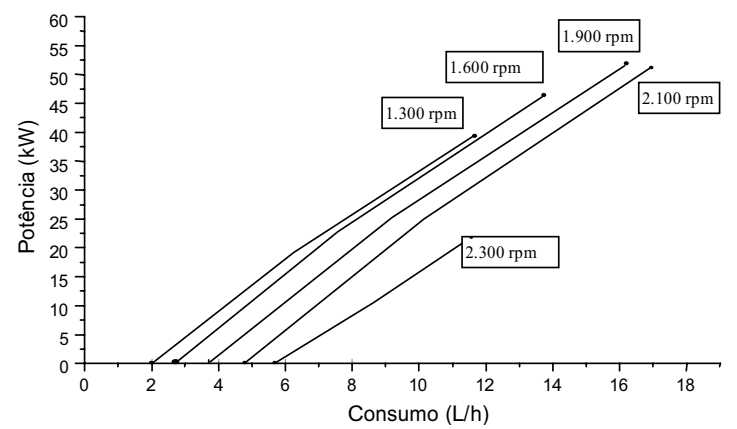

FIG. 1. Curvas de calibração de um trator Massey Ferguson 292 turbo: consumo de combustível $(\mathrm{L} / \mathrm{h}) \times$ potência $(\mathrm{kW})$. Embrapa-CNPMS, Sete Lagoas, MG, 1995. usado permite autonomia de trabalho para mais de uma hora e meia.

O conjunto de aquisição de dados é composto de várias partes, e foi alocado de modo a proteger cabos, conexões e o coletor de dados ("data-logger"). O coletor de dados adotado para este trabalho é o modelo CR10, da Campbell Scientific Ltd. O coletor possui a capacidade de programação, mesmo no campo, por meio de um teclado com visor alfa numérico, que, além de auxiliar na programação, também possibilita o monitoramento dos dados no tempo real. Esse teclado é ligado ao coletor de dados por meio de cabo e conector de 9 pinos. Na configuração utilizada possui 6 entradas de sensores analógicos diferenciais ou 12 analógicos simples e 2 sensores lógicos (contadores), simultaneamente.

O seu funcionamento é baseado em um microcomprocessador que permite a programação do processo de leitura dos sensores e dos campos de armazenamento das informações. O programa desenvolvido neste trabalho foi escrito, inicialmente, em um computador portátil, e transferido ao CR10 via interface serial. No campo, utilizou-se o teclado próprio para pequenos programas de teste ou para monitorar os dados coletados. Utilizou-se, no programa desenvolvido, funções de multiplicações de coeficientes em tempo real durante a leitura, convertendo os dados lidos do sensor em unidades de medida, ou efetuar uma correção do zero ("offset"). Algumas funções de cálculos estatísticos em tempo real mostraram-se bastante úteis no desenvolvimento do programa. O programa foi escrito para que os dados fossem armazenados numa área de memória, e, após o experimento, serem transferidos para o computador portátil. Essa memória é do tipo pilha, e observa-se que para uma aquisição em intervalos menores ao adotado havia a possibilidade de perder os primeiros dados gravados. No sistema, utilizou-se a configuração de memória de 29 kBytes para armazenamento. O sistema ainda permite uma taxa de aquisição de dados que pode variar entre $1 / 64$ e 8.191 segundos.

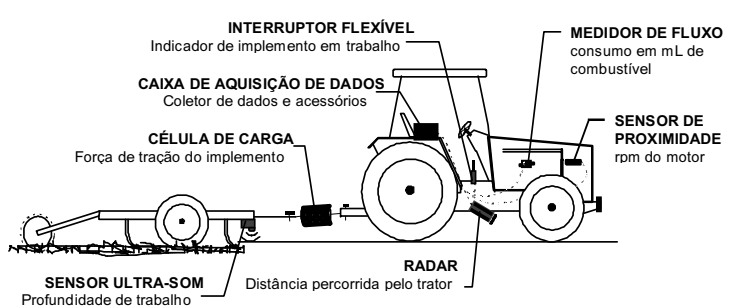

FIG. 2. Sistema de aquisição de dados e posicionamento dos elementos no conjunto trator-implemento.

Pesq. agropec. bras., Brasília, v.34, n.7, p.1241-1246, jul. 1999 
Os sensores utilizados são divididos em dois grupos: analógico e digital (ou lógico). Os sensores analógicos emitem um sinal contínuo de amplitude proporcional à variável de medida. Pelo fato de ser instantânea, no momento da observação, os sensores não usam a informação contida entre duas observações. Os sensores lógicos trabalham digitalmente (aberto ou fechado), ou seja, cada impulso corresponde a uma quantidade precisa do valor medido. Neste caso, a informação não é instantânea, e vai contabilizar todos os impulsos desde a última observação.

Célula de carga: do tipo analógico, modelo CCI da Kratos, com capacidade de $98 \mathrm{kN}$, é baseada em extensômetros dispostos em ponte de "Wheatstone". Os extensiômetros são resistências elétricas sensíveis à deformação. Posicionado entre o trator e o implemento, junto à barra de tração, este sensor mede a força de tração resultante.

Sensor ultra-som: é um sensor para medir distância, do tipo analógico, modelo US 177, da Sick Optique Electronique (França). Mediante transdutores de ultra-som, é efetuada a medida da profundidade de trabalho do implemento. O sinal de saída é analógico, e a tensão é proporcional à distância medida. Conhecendo-se a distância do sensor até o chão, antes do trabalho, a diferença entre essa distância e a distância medida pelo sensor pode ser determinada a profundidade do trabalho.

Interruptor flexível: é um sensor do tipo chave lógico, instalado na alavanca de comando hidráulico dos implementos, e que informa a posição do implemento (transporte ou trabalho). Dessa maneira, é possível diferenciar os dados armazenados durante o trabalho e os coletados nas manobras.

Medidores de fluxo: o medidor de fluxo é um sensor lógico, modelo LS 4150, da Micro Oval II (Japão), que funciona da seguinte maneira: são duas engrenagens ovais; uma delas é marcada por um ímã que sensibiliza sensor indutivo a cada volta ( $1 \mathrm{~mL}$ de volume deslocado). Para esse tipo de medição, são usados dois sensores: um, para medir a quantidade de combustível que entra na bomba, e o outro, mede o retorno depois de passar pelos injetores. Da diferença dos dois resulta o consumo de combustível do trator.

Sensor de proximidade: este sensor é do tipo indutivo lógico, da Instrumentation (França) e está instalado à frente da polia do motor, para medir sua rotação. A cada volta da polia, um pulso é registrado no coletor de dados.

Radar: o sistema de funcionamento é o mesmo utilizado pelo sensor ultra-som, mas as ondas de ultra-som são substituídas pelas de microondas. É um sensor lógico, modelo DJ RVS2, da Dickey John, que gera um pulso elétrico a cada centímetro. Esse sensor foi utilizado para medir a distância percorrida pelo trator em relação à unidade de tempo, e, portanto, a velocidade de deslocamento do trator.

\section{Processamentos dos dados}

Após a coleta dos dados no campo, os dados são transferidos para um computador, a fim de serem processados. Utilizou-se o próprio programa de transferência do "datalogger", que permite escolher a porta de comunicação, a velocidade de transmissão e, também, o gerenciamento da comunicação. Os dados são armazenados no formato de arquivo texto. A Tabela 1 mostra os dados medidos e os sensores associados.

O esquema da Fig. 3 mostra a distribuição das diferentes alternativas de uso da potência no trator, e as suas perdas, para facilitar o entendimento de como foram estimadas.

\section{RESULTADOS E DISCUSSÃO}

\section{Curvas de calibração do motor}

As curvas de calibração do motor, de um trator Massey Ferguson 292 turbo, são apresentadas na Fig. 1, para auxiliar no cálculo indireto da potência, conforme metodologia descrita anteriormente. Como pode ser visto na Fig. 1, a curva de calibração do motor foi feita em uma ampla faixa de rotação, de 1.300 a $2.300 \mathrm{rpm}$, com a finalidade de possibilitar ao usuário trabalhar com o trator dentro da faixa de potência usualmente requerida. Pode-se notar que quando a rotação do motor (rpm) é um valor intermediário aos das curvas de calibração, deve-se fa-

TABELA 1. Relação de sensores e as respectivas medições.

\begin{tabular}{llc}
\hline Sensor & Variável medida & Unidade \\
\hline Radar & Distância percorrida & $\mathrm{m}$ \\
Proximidade & Rotação motor & $\mathrm{rpm}$ \\
Fluxo & Consumo combustível & $\mathrm{mL}$ \\
Ultra-som & Profundidade de trabalho & $\mathrm{cm}$ \\
Célula de carga & Esforço de tração & $\mathrm{kgf}$ \\
Posição do & & \\
implemento & Interruptor & - \\
\hline
\end{tabular}


zer uma interpolação linear para calcular o valor da potência.

A partir dos dados obtidos na curva de calibração (Fig. 1), pode-se obter as curvas de desempenho do trator, dando ao usuário uma excelente informação para os trabalhos de campo do conjunto tratorimplemento, na escolha da faixa de rotação do motor, como mostra a Fig. 4.

Levando-se em consideração os três pontos mais importantes para a programação do "data-logger" ou coletor de dados caixa : memória disponível, característica do coletor ou "data-logger" e precisão, o tempo de um segundo foi escolhido para a obtenção de dados, nos testes de campo. Para diminuir o tamanho do arquivo de dados e ao mesmo tempo

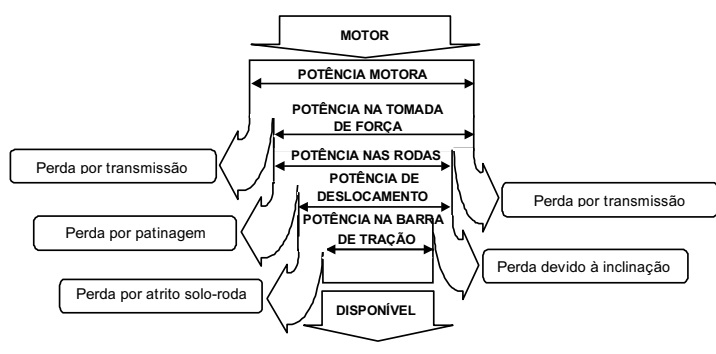

FIG. 3. Esquema da distribuição de uso de potência do trator e suas perdas. Fonte: Pirot $\&$ Vaitilangom (1987).

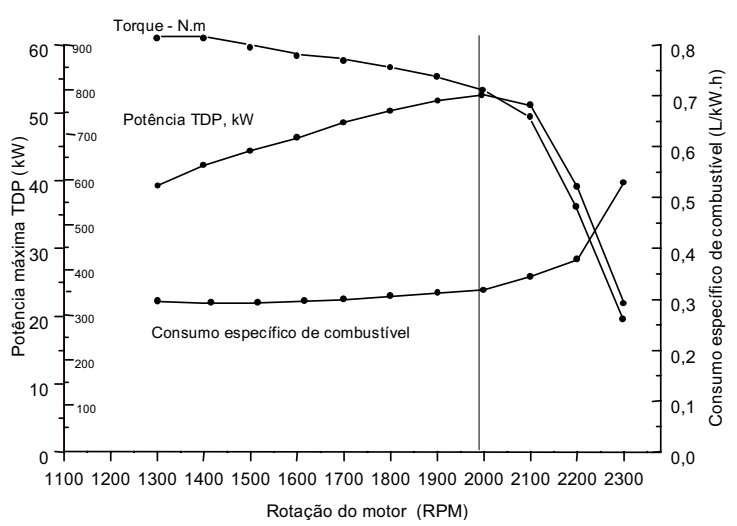

FIG. 4. Desempenho do trator Massey Ferguson -MF 292 Turbo. Embrapa-CNPMS, Sete Lagoas, MG, 1995. evitar erros com os dois tipos de sensores (lógicos e analógicos), os dados são acumulados durante intervalos de 5 segundos e depois o valor médio é gravado na memória final. Dessa forma, o programa usado permite uma autonomia de trabalho para mais de uma hora e meia.

Utilizando-se os dados de rotação do motor e consumo de combustível, das curvas de calibração do trator (Fig. 1), o programa calculou a potência desenvolvida pelo motor. Além disso, a patinagem pode, também, ser calculada, bastando apenas considerar a relação de transmissão da marcha usada durante o trabalho e a rotação do motor.

Finalmente, com os dados calculados acima, associados ao esquema de distribuição de potência (Fig. 4), permitiram obter todos os dados necessários para a avaliação de desempenho do conjunto trator-implemento, durante uma operação de teste de campo, como mostra a Tabela 2.

A partir dos dados organizados e tratados, podese ter a avaliação do desempenho do conjunto trator-implemento, pela montagem de uma tabela, como mostra a Tabela 3 .

A Tabela 3 tem o significado comparativo para análise de desempenho de seu equipamento no campo, através da descrição das características técnicas

TABELA 2. Dados obtidos durante a avaliação com o comboio de ensaios. Embrapa-CNPMS, Sete Lagoas, MG, 1995.

\begin{tabular}{lcr}
\hline Variável & Unidade & $\begin{array}{r}\text { Valor } \\
\text { obtido }\end{array}$ \\
\hline Velocidade & $\mathrm{m} / \mathrm{s}$ & 1,78 \\
Rotação & $\mathrm{rpm}$ & 1788 \\
Consumo & $\mathrm{L} / \mathrm{h}$ & 10,08 \\
Profundidade de trabalho & $\mathrm{cm}$ & 19,37 \\
Patinagem & $\%$ & 9,3 \\
Força de tração medida & $\mathrm{kN}$ & 12,18 \\
Potência na TDP & $\mathrm{kW}$ & 30,0 \\
Potência no eixo & $\mathrm{kW}$ & 28,5 \\
Potência para deslocamento & $\mathrm{kW}$ & 25,8 \\
Potência na barra de tração & $\mathrm{kW}$ & 22,9 \\
Força de tração estimada & $\mathrm{kN}$ & 12,86 \\
\hline
\end{tabular}

Pesq. agropec. bras., Brasília, v.34, n.7, p.1241-1246, jul. 1999 
TABELA 3. Exemplo de um relatório de avaliação de desempenho de tração do conjunto trator-equipamento para preparo de solo.

\begin{tabular}{|c|c|c|c|c|c|c|c|}
\hline \multirow[t]{3}{*}{ Implemento } & \multicolumn{7}{|c|}{ Determinações } \\
\hline & $\begin{array}{c}\text { Teor de umidade } \\
\text { do solo }\end{array}$ & $\begin{array}{c}\text { Potência } \\
\text { na TDP }\end{array}$ & Patinagem & $\begin{array}{l}\text { Consumo } \\
\text { específico }\end{array}$ & $\begin{array}{c}\text { Consumo } \\
\text { horário }\end{array}$ & Velocidade & $\begin{array}{c}\text { Profundidade } \\
\text { de trabalho }\end{array}$ \\
\hline & $(\%)$ & $(\mathrm{kW})$ & $(\%)$ & (L/kW. hora) & (L/hora) & (km/hora) & $(\mathrm{cm})$ \\
\hline Arado escarificador de 5 hastes & 22,5 & 29,7 & 5,8 & 0,41 & 11,7 & 5,7 & 25,0 \\
\hline Arado escarificador de 5 hastes & 31,6 & 28,9 & 6,0 & 0,41 & 10,9 & 5,8 & 24,7 \\
\hline Grade aradora & 31,6 & 22,8 & 2,7 & 0,45 & 9,6 & 7,7 & 7,3 \\
\hline
\end{tabular}

de funcionamento do equipamento e dos resultados da avaliação de desempenho operacional do equipamento. Esses reultados servirão também ao fabricante, para auxiliá-lo quando da necessidade de melhoria do seu produto, e ao agricultor, na racionalização de uso.

\section{CONCLUSÕES}

1. Os testes de desempenho do conjunto tratorimplemento, usando instrumentação eletrônica, permitem que se tenha uma grande capacidade de trabalho, em razão da facilidade de programação e operação da cadeia de medições.

2. A metodologia utilizada nos testes de campo permite obter as curvas de desempenho operacional do conjunto trator-implemento, assim como um relatório de avaliação completo, de forma bastante rápida, o que pode servir de recomendação, tanto para os usuários dos equipamentos, quanto para os fabricantes.

3. A utilização de instrumentação eletrônica para testes de desempenho de equipamentos no campo requer calibração constante, em laboratório, de todos os sensores utilizados na cadeia de medições, para garantir a precisão e a acurácia da medida.

4. Os resultados obtidos do esforço de tração líquida, pela instrumentação eletrônica, no campo, indicam uma tendência de comportamento similar aos comportamentos calculados por meio de fórmulas, com uma correlação de 0,996 .

\section{REFERÊNCIAS}

GAMERO, C.A.; BENEZ, S.H.; FURLAN JÚNIOR, J.A. Análise comparativa de consumo de combustível e da capacidade de campo de diferentes sistemas de preparo periódico do solo. In: CONGRESSO BRASILEIRO DE ENGENHARIA AGRÍCOLA, 15., 1986, São Paulo. Anais... São Paulo: Sociedade Brasileira de Engenharia Agrícola, 1986. p.1-9.

LILJEDAHL, J.N.; CARLETON, W.M.; TURQUIST, P.K.; SMITH, D.W. Tractors and their power units. New York: John Wiley, 1979. 485p.

MANTOVANI, E.C. Compactação do solo. Informe Agropecuário, Belo Horizonte, v.13, n.147, p.52-55, 1987.

PERRET, S.; PIROT, R.; BARRET, D.; GALLET, P.; DEURVEILHER, D. Étude et définition d'un appareillage életctronique d'acquisition de données embarqué sur tracteur. Montpellier: CIRAD-SAR, 1994. 35p. (CIRAD-SAR, n.06/94).

PIROT, R.; VAITILANGOM, G. Étude, réalisation et mise au point d'une chaîne de mesure embarquée pour machines agricoles. Conception des protocoles d'acquisition, de transfert et d'exploitation des données. Machinisme Agricole Tropical, Antony, n.97, p.33-36, 1987. 